\title{
Influence du gauchissement de semis d'épinette noire (Picea mariana (Mill.) B.S.P.) produits en serre sur leur performance en plantation
}

\author{
par Martin Béland et Jean Lapierre
}

\begin{abstract}
Le gauchissement est un défaut de croissance dans la production en serre de semis d'épinette noire qui se manifeste par un affaissement de la tige ou d'une partie de celle-ci. À mesure que se fait la lignification, le semis se redresse mais non sans garder une déformation plus ou moins prononcée. L'objectif de cette étude est d'établir si le gauchissement a un effet permanent sur la croissance et la forme des semis d'épinette noire afin de déterminer dans quelle mesure le rejet des plants gauchis est justifié. Le projet, réalisé en Abitibi (Québec), a consisté en la plantation de 2304 semis d'épinette noire, sur trois sites aux conditions de sols différentes. Les plants ont été classifiés en serre en six classes de gauchissement de gravités différentes incluant un témoin et disposés par blocs aléatoires complets. De 1986, année de la plantation, à 1990 dernière année de mesure, le gauchissement a eu un impact minime sur leur croissance en hauteur et en diamètre. Cependant, lorsque plantés dans un milieu peu propice, certains plants fortement gauchis ont eu une mortalité suffisamment élevée pour justifier de les déclasser. De plus, ils ont développé une cime fourchue dans une plus forte proportion. Cette étude permet de mettre en doute la nécéssité de rejeter systématiquement tous les plants qui ont des déformations plus légères.
\end{abstract}

Mots-clés: Gauchissement, semis, plantation, croissance juvénile, déformation, épinette noire, serre.
Spiralization is a developmental deformation in winter greenhouse produced black spruce seedlings characterized by a drooping stem or portion of stem. During lignification, the seedling redresses but maintains part of its deformation. The objective of this study is to evaluate the long-term effect of spiralization on the growth, survival and morphology of black spruce seedlings in order to determine to what degree the rejection of spiralized seedlings is justified. Field evaluation was undertaken on 2304 black spruce seedlings planted on three sites with different soil conditions in Abitibi, Québec. The seedlings were divided in the greenhouse into six stem deformation classes of varying intensities, including a control, and laid out on a randomized complete block design. From 1986, the year of plantation, to 1990, spiralization had little effect on height and diameter growth. However, severely deformed seedlings planted on a heavy-textured site suffered sufficiently high mortality rates to justify their rejection. Moreover, these seedlings developed a higher proportion of double leaders. The study suggests that systematic rejection of all classes of deformed seedlings, especially those in the less severe classes, is probably not justified.

Key words: Spiralization, stem deformation, seedlings, planting, black spruce, greenhouse, early growth, mortality.

\section{Introduction}

Le gauchissement de la tige, parfois indûment appelé étiolement, est un défaut de croissance qui se manifeste par un affaissement partielle du semis pendant sa phase de croissance exponentielle. Selon Margolis et Lord (1990) et Margolis et al. (1991), ce problème se retrouve surtout sur les semis d'épinette noire (Picea mariana (Mill.) B.S.P.) en récipients, produits en serre pendant l'hiver. Les travaux de ces derniers ont démontré que le phénomène est associé à la diminution du taux de transformation d'un acide aminé, la phénylalanine, en un composé précurseur de la lignine. De plus, il a été démontré que la combinaison des traitements de forte intensité lumineuse et d'oxygénation du substrat racinaire augmentait la lignification des semis d'épinette noire. Selon Thompson (1985), les courbures à la base des tiges ("basal sweep"), sont un phénomène différent du gauchissement et sont plutôt dues à de mauvaises transplantations.

Un semis gauchi est le plus souvent déclassé et rejeté par le Ministère des Forêts du Québec (MFO). En effet le guide d'évaluation de la qualité des plants (Brouillette et al. 1988) recommande de rejeter les plants lorsque la tige est inclinée de plus de $15^{\circ}$ par rapport à la verticale ou lorsque la tige est fortement déformée. Si l'on considère que le gauchisse-

Unité de recherche et de développement forestiers de l'AbitibiTémiscamingue, Université du Québec en Abitibi-Témiscamingue, $42 \mathrm{Mgr}$ Rhéaume Est, case postale 700, Rouyn-Noranda (Québec), J9X 5E4 ment peut affecter de 5 à $20 \%$ d'une production d'épinettes noires, ceci résulte en une perte financière substantielle pour les entreprises de production. D'un autre côté, selon Binder et al. (1988), la plantation d'un semis de piètre qualité peut coûter trois fois plus que la destruction par erreur d'un semis acceptable, ceci sans même considérer les coûts pour replanter ou les intérêts de ces coûts.

Après un certain temps, les plants se redressent, parfois à un point tel qu'une partie d'entre eux puissent être jugés acceptables pour le reboisement. À partir de cette constatation, l'objectif de cette étude est donc d'établir si le gauchissement a un effet sur la survie, la croissance en hauteur et en diamètre, la forme des tiges d'épinette noire et le développement de cimes fourchues ou multiples afin de déterminer dans quelle mesure le rejet des plants gauchis est justifié.

\section{Matériel et méthode}

Provenance des graines, régie de culture et classes de gauchissement

Les plants ont été produits aux Serres coopératives de Guyenne $\left(48^{\circ} 45^{\circ} \mathrm{N}, 78^{\circ} 40^{\circ} \mathrm{O}\right)$ à partir de graines d'épinettes noires de deux provenances ou de deux zones. Ce sont les zones " 1 " et " 4 " de récolte de cônes du MFO. La zone " 1 "' s'étend d'est en ouest à travers la région jusqu'à environ $49^{\circ} 30^{\prime} \mathrm{N}$, au nord, et jusqu'à $48^{\circ} 10^{\prime} \mathrm{N}$, au sud, 


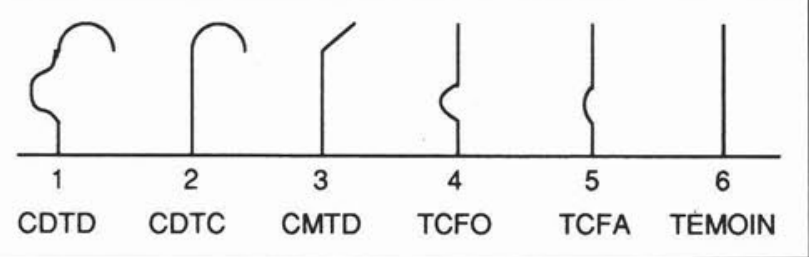

Figure 1. Schéma des six classes de gauchissement retenues.

en incluant les serres et les plantations à l'étude. La zone " 4 " est adjacente au sud de la zone " 1 " et s'étend jusqu'à $47^{\circ} 10^{\prime} \mathrm{N}$.

Les graines ont été ensemencées dans des "multipots" à la mi-décembre 1985. Après trois mois de croissance avec photopériode de 18 heures, les plants ont été classifiés selon les six catégories de gauchissement suivantes (Fig. 1), choisies avec l'aide des fonctionnaires du bureau régional du MFO.

CDTC - Plants ayant une cime descendante et une tige croche;

CDTD - Plants ayant une cime descendante et une tige droite;

CMTD - Plants ayant une cime montante mais dont l'angle par rapport à la verticale est supérieur à $15^{\circ}$ et dont la tige est droite;

TCFO - Plants ayant une cime verticale mais une tige avec courbe forte;

TCFA - Plants ayant une cime verticale mais une tige avec courbe faible;

TÉMOIN - Plants témoins droits.
Vers la fin-avril et le début-mai, les plants ont subit un traitement de jours courts (8-9 heures) afin d'initier la dormance et ont été plantés les 11 et 12 juillet 1986 .

\section{Dispositif expérimental}

Le dispositif expérimental utilisé est reproduit sur trois sites différents dans le canton de Guyenne, Abitibi (Fig. 2).

Le premier se situe sur un sol argileux imparfaitement drainé qui a été déboisé il y a plusieurs années, mais non cultivé. Ce site est couvert principalement de peupliers fauxtrembles qui ont dû être dégagés sur une superficie suffisante pour le projet.

Le second est situé sur un sol sablonneux couvert d'un peuplement épars de pin gris d'une trentaine d'années, originant d'un feu. Un hectare de ce peuplement a été coupé pour recevoir le dispositif.

Le troisième est un terrain agricole sur un sol organique mince qui a subit des travaux de drainage. On y a déjà produit des légumes mais le terrain aux alentours ne sert plus que pour le foin.

La première année, un désherbage chimique a dû être entrepris sur le site argileux tandis que les années suivantes, un désherbage manuel a été suffisant. Un désherbage manuel des graminées a aussi été entrepris à chaque année sur le site organique. Le peu de végétation compétitrice sur le site sablonneux n'a pas justifié de traitement.

L'échantillonnage est réalisé par blocs aléatoires complets. Sur chaque site on retrouve quatre blocs contenant douze parcelles correspondant à la combinaison des deux provenances et des six classes de déformation (Fig. 3). Les parcelles comprennent chacune 16 plants d'une même provenance et d'une même classe de déformation, pour un total de 2304 plants

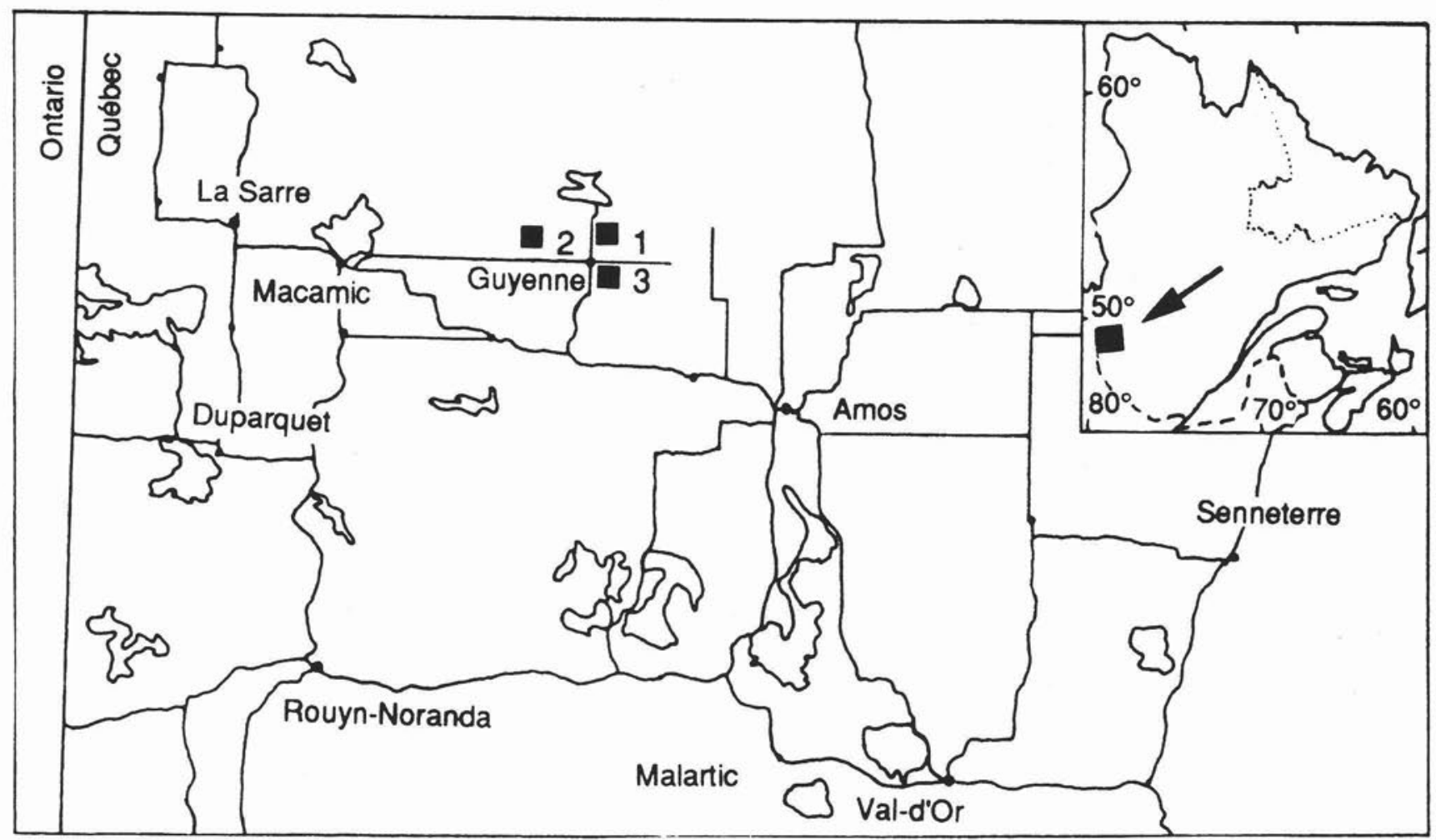

Figure 2. Localisation du territoire d'étude. 


\begin{tabular}{|c|c|c|c|c|c|}
\hline & $\begin{array}{c}\text { Parcelle } \\
2\end{array}$ & $\begin{array}{c}\text { Parcelle } \\
3\end{array}$ & $\begin{array}{c}\text { Parcelle } \\
4\end{array}$ & $\begin{array}{c}\text { Parcelle } \\
5\end{array}$ & $\begin{array}{c}\text { Parcelle } \\
6\end{array}$ \\
\hline $\begin{array}{c}\text { Parcelle } \\
7\end{array}$ & $\begin{array}{c}\text { Parcelle } \\
8\end{array}$ & $\begin{array}{c}\text { Parcelle } \\
9\end{array}$ & $\begin{array}{c}\text { Parcelle } \\
10\end{array}$ & $\begin{array}{c}\text { Parcelle } \\
11\end{array}$ & $\begin{array}{c}\text { Parcelle } \\
12\end{array}$ \\
\hline
\end{tabular}

BLOC IV

Figure 3. Schéma du dispositif expérimental répété sur chacun des trois sites.

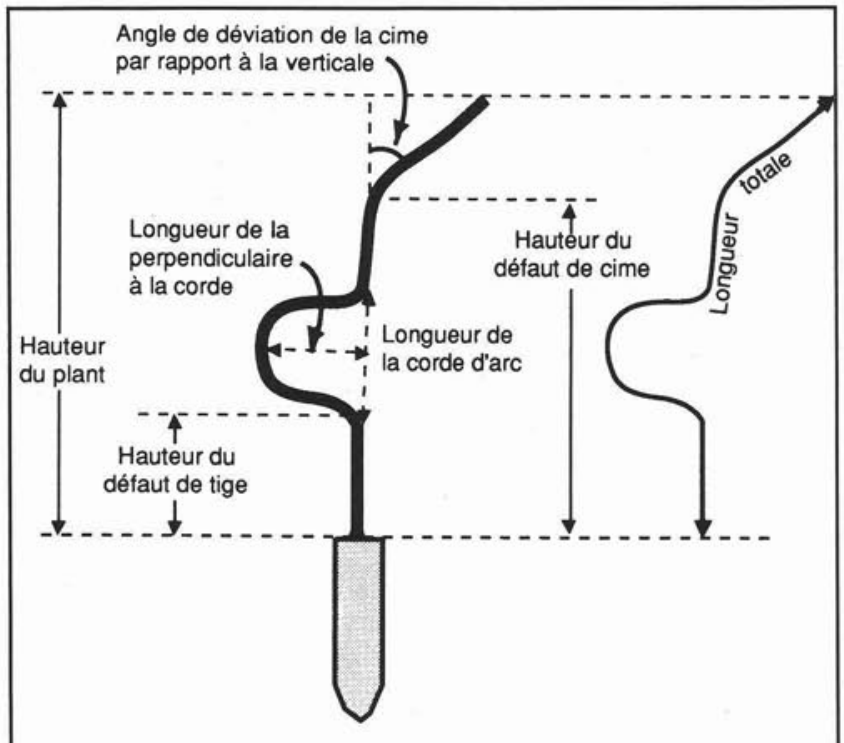

Figure 4. Mesures prises lors de la mise en terre des plants en 1986.

mis en terre, espacés de 2 mètres par 2 mètres. Des plants ont été ajoutés en périphérie pour éliminer l'effet de bordure. La plantation a eu lieu les 11 et 12 juillet 1986.

\section{Mesures}

Avant la mise en terre, la morphologie des plants a été mesurée en détail (Fig. 4). Ces mesures comprenaient la longueur totale, la hauteur des défauts de cime et de tige s'il y a lieu ainsi que l'angle de déviation de la cime par rapport
Tableau 1. Pourcentage de survie en 1990 par classe de déformation pour chaque combinaison de site et de provenance.

\begin{tabular}{lcrrrrr}
\hline \multirow{2}{*}{$\begin{array}{l}\text { Site et } \\
\text { prov. }\end{array}$} & \multicolumn{6}{c}{ Classe de déformation } \\
\cline { 2 - 7 } & CDTC & CDTD & CMTD & TCFO & TCFA & TÉMOIN \\
\hline $\begin{array}{l}\text { Argile } \\
\text { prov. 1 }\end{array}$ & $-25,00$ & 39,06 & 56,25 & 64,06 & 51,56 & 56,25 \\
$\begin{array}{l}\text { Argile } \\
\text { prov. 4 }\end{array}$ & $-26,56-32,81$ & 37,50 & 54,69 & 46,88 & 51,56 \\
$\begin{array}{l}\text { Sable } \\
\text { prov. 1 }\end{array}$ & $-71,88-71,88$ & 90,63 & $87,50-79,69$ & 93,75 \\
$\begin{array}{l}\text { Sable } \\
\text { prov. 4 }\end{array}$ & $-68,75-75,00$ & $87,50-71,88$ & 85,94 & 92,19 \\
$\begin{array}{l}\text { Organique } \\
\text { prov. 1 }\end{array}$ & $-87,50-87,50-82,81-84,38$ & 93,75 & 96,88 \\
$\begin{array}{l}\text { Organique } \\
\text { prov. 4 }\end{array}$ & 76,56 & $84,38+90,63$ & 78,13 & 84,38 & 75,00 \\
\hline
\end{tabular}

'Les signes " +" et "- " indiquent une différence significative (positive et négative respectivement) entre la classe de déformation et le témoin.

à la verticale et l'amplitude du défaut de tige en mesurant la longueur de la corde de cette courbure et la longueur de la perpendiculaire entre l'arc et la corde (Fig. 4). À ces mesures s'ajoutaient la hauteur et le diamètre au collet de chaque plant.

À la fin de l'été de chacune des quatre années subséquentes, la hauteur et le diamètre des plants ont été remesurés et un plant par parcelle (144 plants au total) a fait l'objet d'un suivi photographique. Enfin, lors de la dernière année de mesure, la présence de cimes fourchues et de cimes multiples furent notées.

\section{Analyses statistiques}

Les moyennes des hauteurs, diamètres, croissances en hauteur et en diamètre depuis 1986 des plants gauchis ont été comparées à celles des plants témoins à l'aide d'une analyse de variance et d'une comparaison des moyennes des moindres carrés (Scherrer 1984). Aucun effet bloc n'a été relevé, mais les interactions significatives entre le site, la provenance et la classe de déformation ont nécessité d'analyser chaque combinaison séparément. La normalité des données, vérifiée à l'aide du test de Wilk-Shapiro et du test D de Kolmogorov (Scherrer 1984), a été jugée satisfaisante et le test de Bartlett a révélé l'homogénéité des variances des données. Les taux de mortalité, l'occurrence de cimes fourchues et de cimes multiples ont été comparés à l'aide du test $\mathrm{G}$ de comparaisons multiples de pourcentages (Scherrer 1984). Le seuil de signification considéré pour l'analyse de variance et les tests de comparaisons multiples est de 0,05.

Enfin, les corrélations entre les mesures détaillés de la morphologie des plants prises en 1986 et la croissance en hauteur et en diamètre ont été mesurées avec le coefficient de Pearson (Scherrer 1984).

\section{Résultats et discussion}

\section{Survie en 1990}

Pendant les quatre années de croissance au champ sur le site argileux, de très fortes mortalités ont été observées (jusqu'à $70 \%$ ). Outre les caractéristiques du sol proprement dites, cela semble dû à plusieurs facteurs dont le broutage par le lièvre, le mauvais drainage et le décapage de la couche 


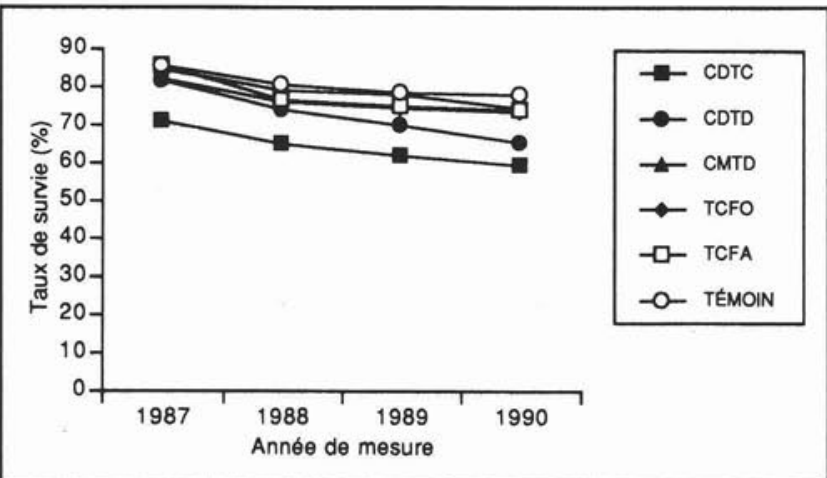

Figure 5. Évolution de la survie par classe de déformation pendant les quatre années de croissance en plantation, pour l'ensemble des plants.

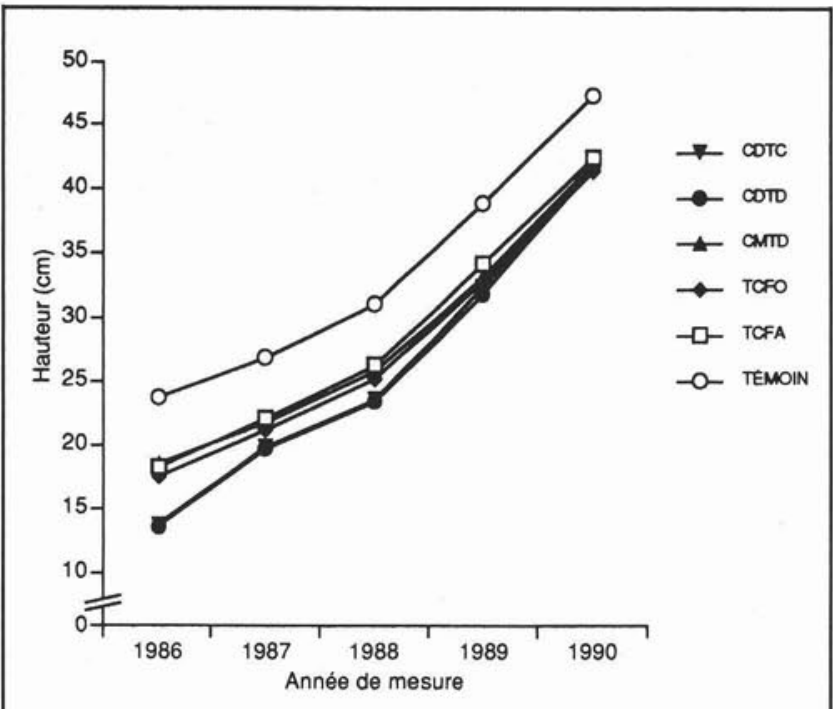

Figure 6. Hauteurs moyennes des plants par classe de déformation pour chaque année de mesures et sur l'ensemble des données.

organique par endroit suite à la préparation de terrain. Toutefois, ces conditions difficiles devraient avoir eu une influence semblable sur tous les plants de ce site, peu importe le type de déformation de la tige. Enfin, les effectifs de plants résultants en 1990 sont suffisants pour en tirer des résultats valables.

La survie des plants à cime descendante et à tige croche (CDTC) est dans tous les cas (sauf sur sol organique et provenance 4) inférieure à celle des plants témoins (tableau 1). Celle des plants à cime descendante et à tige droite (CDTD) est aussi dans la plupart des cas inférieure à celle des plants témoins. Pour ce qui est des plants à cime montante et tige droite (CMTD), des plants à tige fortement courbée (TCFO) et des plants à tige faiblement courbée (TCFA), leur survie n'est pas, sauf exception, inférieure à celle des plants témoins. Ces résultats sont bien illustrés par la Fig. 5 où l'ensemble des sites et des provenances sont regroupés. On y constate aussi que ces tendances sont cohérentes avec les résultats de 1987, 1988 et 1989 et que la mortalité semble diminuer.

Les plants de certaines classes de déformation, principalement les plants à cime descendante, ont subit dans plusieurs
Tableau 2. Croissance moyenne (cm) en hauteur de 1986 à 1990 par classe de déformation pour chaque combinaison de site et de provenance.

\begin{tabular}{lrrrrrr}
\hline \multirow{2}{*}{$\begin{array}{l}\text { Site et } \\
\text { prov. }\end{array}$} & \multicolumn{6}{c}{ Classe de déformation' } \\
\cline { 2 - 7 } & CDTC & CDTD & CMTD & TCFO & TCFA & TÉMOIN \\
\hline $\begin{array}{l}\text { Argile } \\
\text { prov. 1 }\end{array}$ & 13,99 & 23,12 & $-12,12$ & 17,67 & 15,47 & 18,56 \\
$\begin{array}{l}\text { Argile } \\
\text { prov. 4 }\end{array}$ & $+19,24+19,27$ & 14,21 & 14,34 & 13,17 & 9,96 \\
$\begin{array}{l}\text { Sable } \\
\text { prov. 1 }\end{array}$ & 15,51 & 19,38 & 20,31 & 15,83 & 20,09 & 18,09 \\
$\begin{array}{l}\text { Sable } \\
\text { prov. 4 }\end{array}$ & 18,07 & 15,77 & $14,94+19,95$ & 14,52 & 16,01 \\
$\begin{array}{l}\text { Organique } \\
\text { prov. 1 }\end{array}$ & 42,39 & 40,35 & 37,72 & $38,21-32,39$ & 41,20 \\
$\begin{array}{l}\text { Organique } \\
\text { prov. 4 }\end{array}$ & $+40,81+38,63$ & 32,15 & $31,15+40,91$ & 31,79 \\
\hline
\end{tabular}

"Les signes "+" et "-_" indiquent une différence significative (positive et négative respectivement) entre la classe de déformation et le témoin.

cas de plus fortes mortalités. Cependant, dans d'autres cas, tel que ce qui a été rencontré sur sol organique, avec la provenance 4 , tous les plants ont survécu aussi bien que les plants témoins.

\section{Hauteur}

Les mesures de hauteur et de diamètre en 1990 auraient pu être analysées telles quelles. Cependant, comme le suggèrent Brand et al. (1987), il est souvent très pertinent d'utiliser des mesures de croissance relative pour des expériences répétées sur plusieurs années avec des arbres. Pour cette raison, l'accroissement entre 1986 et 1990 sera aussi discuté.

Autant l'année de la plantation que lors des quatre années de mesures subséquentes, la hauteur des plants témoins non gauchis était supérieure à celle de l'ensemble des plants gauchis (Fig. 6). De plus, dans l'ensemble, les plants gauchis n'ont pas rattrapé le retard qu'ils avaient dès le départ, mais ont plutôt suivi une évolution presque parallèle aux plants témoins.

Ces tendances sont confirmées, au tableau 2, lorsqu'on constate qu'il y a très peu de situation où les plants témoins ont un avantage sur les plants gauchis au niveau de la croissance en hauteur. La croissance en hauteur étant comprise comme la différence entre la hauteur en 1990 et en 1986. Il y a même des cas où cette croissance est légèrement supérieure chez les plants gauchis, ce qui laisse croire que les retards de croissance en hauteur encore observables en 1990 , de l'ordre de $5 \mathrm{~cm}$, pourraient devenir négligeables avec les années.

\section{Diamètre}

De la même manière que pour la hauteur, autant l'année de la plantation que lors des quatre années de mesures subséquentes, le diamètre au collet des plants témoins non gauchis était supérieure à celui de l'ensemble des plants gauchis (Fig. 7). De plus, dans l'ensemble, les plants gauchis n'ont pas rattrapé le retard qu'ils avaient dès le départ, mais ont plutôt suivi une évolution presque parallèle aux plants témoins (Fig. 7).

Ces tendances sont confirmées, au tableau 3, lorsqu'on constate qu'il y a très peu de situations où les plants témoins ont un avantage sur les plants gauchis au niveau de la 


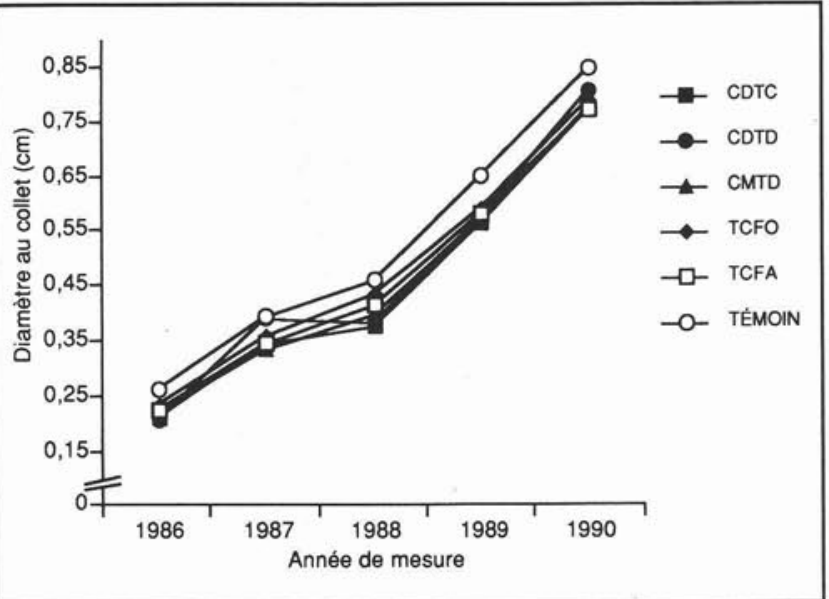

Figure 7. Diamètres au collet moyens des plants par classe de déformation pour les cinq années de mesure, sur l'ensemble des données.

Tableau 3. Croissance moyenne $(\mathrm{cm})$ en diamètre au collet en 1990 par classe de déformation pour chaque combinaison de site et de provenance.

\begin{tabular}{lrrrrrrr}
\hline \multirow{2}{*}{$\begin{array}{l}\text { Site et } \\
\text { prov. }\end{array}$} & \multicolumn{6}{c}{ Classe de déformation } \\
\cline { 2 - 7 } & CDTC & CDTD & CMTD & TCFO & TCFA & TÉMOIN \\
\hline $\begin{array}{l}\text { Argile } \\
\text { prov. 1 }\end{array}$ & $-0,30$ & 0,60 & $-0,41$ & 0,51 & 0,51 & 0,56 \\
$\begin{array}{l}\text { Argile } \\
\text { prov. 4 }\end{array}$ & 0,41 & 0,48 & 0,48 & 0,38 & 0,41 & 0,45 \\
$\begin{array}{l}\text { Sable } \\
\text { prov. 1 }\end{array}$ & $-0,41$ & 0,52 & 0,54 & 0,48 & 0,53 & 0,53 \\
$\begin{array}{l}\text { Sable } \\
\text { prov. 4 }\end{array}$ & 0,53 & 0,43 & 0,45 & 0,49 & 0,44 & 0,50 \\
$\begin{array}{l}\text { Organique } \\
\text { prov. 1 }\end{array}$ & 0,71 & 0,74 & 0,72 & 0,71 & $-0,62$ & 0,80 \\
$\begin{array}{l}\text { Organique } \\
\text { prov. 4 }\end{array}$ & 0,69 & $+0,71$ & 0,65 & $+0,71$ & 0,69 & 0,61 \\
\hline
\end{tabular}

"Les signes "+" et "-" indiquent une différence significative (positive et négative respectivement) entre la classe de déformation et le témoin.

Tableau 4. Coefficients de corrélation de Pearson entre la croissance et les mesures prises sur les plants en $1986^{1}$.

\begin{tabular}{|c|c|c|c|c|c|}
\hline $\begin{array}{l}\text { Variable } \\
\text { corrélée }\end{array}$ & & $\begin{array}{c}\text { Corrélation } \\
\text { avec la } \\
\text { croissance en } \\
\text { hauteur }\end{array}$ & Effectifs & $\begin{array}{l}\text { Corrélation } \\
\text { avec la } \\
\text { croissance en } \\
\text { diamètre }\end{array}$ & Effectifs \\
\hline $\begin{array}{l}\text { Longueur totale } \\
\text { du plant }\end{array}$ & * & $-0,16758$ & 1328 & $-0,05294$ & 1324 \\
\hline $\begin{array}{l}\text { Hauteur du défaut } \\
\text { de cime }\end{array}$ & * & $-0,17591$ & 763 & $*-0,07898$ & 761 \\
\hline $\begin{array}{l}\text { Hauteur du défaut } \\
\text { de tige }\end{array}$ & & $-0,00532$ & 793 & 0,04729 & 791 \\
\hline $\begin{array}{l}\text { Angle de } \\
\text { déviation }\end{array}$ & & 0,10091 & 285 & 0,06831 & 283 \\
\hline $\begin{array}{l}\text { Longueur de la } \\
\text { corde d'arc }\end{array}$ & & $-0,06546$ & 510 & 0,01191 & 508 \\
\hline $\begin{array}{l}\text { Longueur de la } \\
\text { perpendiculaire } \\
\text { à la corde }\end{array}$ & & $-0,08111$ & 510 & $-0,01529$ & 508 \\
\hline
\end{tabular}

${ }^{1}$ Les astérisques indiquent une corrélation significative.
Tableau 5. Pourcentage en 1990 de plants ayant développé une cime multiple par classe de déformation pour chaque combinaison de site et de provenance.

\begin{tabular}{lrrrrrrr}
\hline \multirow{2}{*}{$\begin{array}{l}\text { Site et } \\
\text { prov. }\end{array}$} & \multicolumn{6}{c}{ Classe de déformation } \\
\cline { 2 - 7 } & CDTC & CDTD & CMTD & TCFO & TCFA & TÉMOIN \\
\hline $\begin{array}{l}\text { Argile } \\
\text { prov. 1 }\end{array}$ & 31,25 & 16,00 & 25,00 & 26,83 & 24,24 & 27,78 \\
$\begin{array}{l}\text { Argile } \\
\text { prov. 4 }\end{array}$ & $-5,88$ & 23,81 & 29,17 & 11,43 & 26,67 & 32,35 \\
$\begin{array}{l}\text { Sable } \\
\text { prov. 1 }\end{array}$ & 17,02 & 23,91 & 18,97 & 17,86 & 13,73 & 16,67 \\
$\begin{array}{l}\text { Sable } \\
\text { prov. 4 }\end{array}$ & 29,55 & 22,92 & 19,64 & 30,43 & 36,36 & 33,90 \\
$\begin{array}{l}\text { Organique } \\
\text { prov. 1 }\end{array}$ & 8,93 & 8,77 & 7,55 & 9,26 & 8,33 & 9,68 \\
$\begin{array}{l}\text { Organique } \\
\text { prov. 4 }\end{array}$ & 6,12 & 5,56 & 6,90 & 8,00 & 9,26 & 4,17 \\
\hline
\end{tabular}

'Les signes "-_" indiquent une différence significative (négative) entre la classe de déformation et le témoin.

croissance en diamètre. Il y a même des cas où cette croissance est légèrement supérieure chez les plants gauchis, ce qui laisse croire que les retards de croissance en diamètre encore observables en 1990, de l'ordre de $1 \mathrm{~mm}$, pourraient aussi devenir négligeables avec les années.

\section{Mesures détaillées de la morphologie des plants}

Ces mesures ont été prises afin d'évaluer si des mesures plus détaillées de la déformation des plants feraient ressortir davantage l'influence du gauchissement sur la croissance que la classification utilisée (Fig. 1). Les résultats du tableau 4 indiquent que la croissance en hauteur n'a été significativement corrélée qu'avec la longueur totale et avec la hauteur du défaut de cime. La croissance en diamètre n'a été corrélée significativement qu'avec la hauteur du défaut de cime. Il faut préciser que ces mesures n'ont pu être prises que sur les classes de déformation auxquelles elles s'appliquent, ce qui explique les effectifs réduits. De plus, dans chacun de ces cas, les coefficients sont si faibles qu'ils ne semblent pas fournir un meilleur indice de l'influence du gauchissement.

\section{Évolution des déformations}

La Commission national de foresterie de Finlande recommande que les semis en récipients ne soient pas fourchus et n'aient pas de cimes multiples (Puttonen 1985).

À certains égards, les semis à cimes multiples présentent des caractéristiques semblables à ceux qui sont gauchis: possibilité de retard de croissance et possibilité de malformation permanente de la tige. Les dommages aux méristèmes terminaux et la perte subséquente de dominance apicale causant l'apparition de cimes multiples peuvent survenir durant la formation des bourgeons en présence de résidus d'herbicides ou bien suite au gel et à la dessication hivernale des bourgeons (Webb et Reese 1984). Selon Thompson (1985), les cimes multiples peuvent aussi être d'origine génétique ou bien être dues au broutage ou à la maladie. Dans ce second cas, les semis reprennent rapidement le dessus au champ en produisant une pousse latérale qui reprend la dominance. Durant la première année, la compétition entre les pousses dominantes peut diminuer la croissance. Hofstra et al. (1988), dans une étude sur les semis à cimes multiples 
Tableau 6. Pourcentage en 1990 de plants ayant développé une cime fourchue par classe de déformation pour chaque combinaison de site et de provenance.

\begin{tabular}{|c|c|c|c|c|c|c|}
\hline \multirow{2}{*}{$\begin{array}{l}\text { Site et } \\
\text { prov. }\end{array}$} & \multicolumn{6}{|c|}{ Classe de déformation ${ }^{1}$} \\
\hline & CDTC & CDTD & CMTD & TCFO & TCFA & TÉMOIN \\
\hline $\begin{array}{l}\text { Argile } \\
\text { prov. } 1\end{array}$ & $+50,00$ & 28,00 & $+38,89$ & $+41,46$ & $+36,36$ & 13,89 \\
\hline $\begin{array}{l}\text { Argile } \\
\text { prov. } 4\end{array}$ & 23,53 & 14.29 & 29,17 & 40,00 & 23.33 & 18,18 \\
\hline $\begin{array}{l}\text { Sable } \\
\text { prov. } 1\end{array}$ & $+17,02$ & +21.74 & $+8,62$ & +16.07 & 3,92 & 0,00 \\
\hline $\begin{array}{l}\text { Sable } \\
\text { prov. } 4\end{array}$ & $+22,73$ & 6,25 & 8,93 & 8,70 & 7,27 & 3,39 \\
\hline $\begin{array}{l}\text { Organique } \\
\text { prov. } 1\end{array}$ & $+16,07$ & 8,77 & 9,43 & 12.96 & 8,83 & 4,84 \\
\hline $\begin{array}{l}\text { Organique } \\
\text { prov. } 4\end{array}$ & 14,29 & 12.96 & 6.90 & 8,00 & 18.52 & 16,67 \\
\hline
\end{tabular}

'Les signes " + " indiquent une différence significative (positive) entre la classe de déformation et le témoin.

ont constaté que ces derniers étaient d'une hauteur significativement plus petite mais d'un diamètre au collet semblable aux témoins. Selon Menzies et al. (1985), les fourches ou cimes multiples laissent des séquelles qui persistent en plantation malgré le fait qu'une des cimes devienne dominante sur les autres. Selon Omi et al. (1986), cette dominance devrait apparaître après deux ans de croissance et les effets à long terme des cimes multiples restent à examiner. Ces derniers, dans une étude où $21 \%$ des semis de Pseudotsuga menziesii avaient des cimes multiples, ont trouvé une corrélation négative en entre la fréquence des cimes multiples et la croissance en hauteur de la première année.

Le gauchissement n'a eu, dans la presque totalité des cas, aucun impact sur le développement de cimes multiples (tableau 5), mais a eu un impact certain sur l'occurence de cimes fourchues. En effet, les plants gauchis de provenance 1 sur sol argileux et sur sol sablonneux ont plus souvent développé des cimes fourchues. Il est à noter que les différences obtenues sont parfois très grandes, particulièrement pour les plants à cime descendante et tige croche et pour les plants dont la tige est fortement courbée (tableau 6).

L'observation des photographies a révélé que plusieurs plants semblent avoir eu des problèmes de cimes multiples ou de cimes fourchues dans les premières années de vie en plantation, mais que par la suite, une dominance s'est manifestée parmi les pousses latérales compétitionnant entre elles.

Un éventuel redressement des tiges déformées ne peut être confirmé. À long terme, il est cependant possible que la déformation devienne de moins en moins apparente à mesure que le tronc croîtra en diamètre.

\section{Conclusions}

De manière générale, les résultats de cette étude nous portent à conclure que le gauchissement des semis d'épinettes noires a une influence minime à long terme sur leur croissance en hauteur et en diamètre. Cependant, lorsque certains plants les plus déformés, en l'occurrence les plants à cime descendante (classes "CDTC" et "CDTD") sont plantés en milieu aux conditions plus limitantes (argile), leur survie peut en être affectée. Cette plus forte mortalité, ajoutée à l'incidence accrue des cimes multiples justifie le rejet des plants les plus fortement gauchis. Toutefois, il est permis de mettre sérieusement en doute la nécessité de rejeter systématiquement tous les plants qui ont des déformations plus légères.

L'interprétation des résultats de cette étude doit être réalisée en tenant compte du fait qu'elle a été effectuée sur un nombre limité de sites et de provenances de graines. Ainsi, l'extrapolation des conclusions devra être faite avec prudence.

\section{Remerciements}

Les auteurs souhaitent remercier les Serres Coopératives de Guyenne qui ont produit les plants et fourni du personnel pour la prise annuelle des mesures. Ils souhaitent également remercier Michel Villeneuve et Danielle Charron pour la prise des photographies et Brian Harvey pour ses commentaires sur le manuscrit. Ce projet a été subventionné par les Serres Coopératives de Guyenne, le Ministère des Forêts du Québec et l'Office de la planification et du développement du Québec.

\section{Bibliographie}

Binder, W.D., R.K. Skagel et G.J. Krumlik. 1988. Root growth potential: facts, myths, value? dans: General Technical Report no. 167. Rocky Mountain Forest and Range Experiment Station, USDA Forest Service, paper presented at a meeting of the Western Forest Nursery Association, August 1988, Vernon, British-Columbia.

Brand, D.G., G.F. Weetman et P. Rehsler. 1987. Growth analysis of perennial plants: the relative production rate and its yield components. Annals of Botany. 59: 45-53.

Brouillette, J.G., J.P. Tétreault et J. Lortie. 1988. Guide d'évaluation de la qualité des plants produits en récipients (deuxième version). Service de la régénération forestière, Ministère de l'Énergie et des Ressources du Québec. 47 p.

Hofstra, G., C.M. McLeod et J. Ensing. 1988. Incidence and performance of multiple-leadered seedlings of black and white spruce in Canadian Nurseries. Northern J. Applied Forestry. 5(2): 99-103. Margolis, H.A., S. Delaney, L.-P. Vézina et P. Bellefleur. 1991. Partitioning of ${ }^{14} \mathrm{C}$ between growth and differentiation within stemdeformed and healthy black spruce seedlings. Can. J. Bot. 69: 1225-1231.

Margolis, H.A. et D. Lord. 1990. Deformation of the stem of containerized black spruce seedlings dans: Seedling production in Quebec: Bareroot versus Container Seedlings. Paper read at the Northeastern State, Federal and Provincial Nursurymen's Conference, Montréal, Québec.

Menzies, M.I., J.C. van Dorsser et J.M. Balneaves. 1985. Seedling quality - radiata pine as a case study. Pages 384-415 dans: D.B. South (éd). Proceedings of the International Symposium on Nursery Management Practices for the Southern Pines. Montgomery, Alabama, August 1985, cosponsored by School of Forestry, Alabama Agricultural Experiment Station, Auburn University.

Omi, S.K., G.T. Howe et M.L. Duryea. 1986. First-year field performance of douglas-fir seedlings in relation to nursery characteristics. Pages 29-34 dans: General Technical Report no. 137. Rocky Mountain Forest and Range Experiment Station, USDA Forest Service. Puttonen, P. 1985. Current quality requirements of seedlings in Finland. Pages 557-564 dans: D.B. South (éd.) Proceedings of the International Symposium on Nursery Management Practices for Southern Pines. August 1985, Montgomery, Alabama.

Scherrer, B. 1984. Biostatistique. G. Morin (Ed.). 850 p. Thompson, B.E. 1985. Seedling morphological evaluation - what you can tell by looking. Pages 59-71 dans: Evaluating Seedling Quality: Principles, Procedures, and Predictive Abilities of Major Tests. Proceedings of a workshop held October 1984, Forest Research Laboratory, Oregon State University, Corvallis.

Webb, D.P. et K.H. Reese. 1984. Multiple leadering of coniferous nursery stock. Joint report no. 3. Great Lakes Forest Research Centre and the Ontario Ministry of Natural Resources. 8 p. 